nor the pulse above 108. The wound was dressed for the first time on the tenth day, when it was found to have completely healed, and all the sutures were then withdrawn. The bowels were moved for the first time on the twelfth day by a simple enema, a teaspoonful of castor oil having been given the preceding evening. On April 10th, convalescence, which had up to this time continued uninterruptedly, was complicated by a slight attack of phlegmasia dolens in the left leg, which, however, soon disappeared wander appropriate treatment. The patient left the hospital perfectly well on May 2nd.

\section{DORSET COUNTY HOSPITAL.}

SUCCESSFUL CASE OF PRIMARY AMPUTATION OF RIGHT LEG BELOW THE KNEE, IN A PATIENT AGED SEVENTY-FIVE.

(Under the care of Mr. A. EMson,)

FOR notes of this case we are indebted to Mr. Herbert J. Capon, house-surgeon.

Sophia $S —$, aged seventy-five, was admitted Oct. 30th, 1877. She was a tall, thin, and intelligent woman, and gave the following history. In August, 1874, while ascending some steps with a pail of water, she slipped and bruised her right ankle-joint. She felt something crack, which she supposed was the small bone of her leg. She did not lie up in bed, but continued with her household work as usual, resting when she could. She experienced much pain a little above the inner ankle, which increased, and caused her to seek medical advice in the following March. The painful spot did not seem to improve under local applications, but a crop of pimples appeared, which gradually extended into an open wound, with an elevated base. Caustics were then applied, and in September it was half as large as one's open hand, when she took to her bed. The ulcerated surface extended all around the leg, but the pain was confined to the original spot above the inner ankle. Her foot swelled, and in September, 1877, the bone was exposed. In the beginning of October, while attempting to walk with crutches, one crutch slipped, and with but very slight force her leg was broken across at the seat of the wound. It gave her so little pain that she did not know a fracture had occurred till she observed the end of the leg and foot so freely movable when in bed.

On admission her foot and about three inches of the bones of the leg were doubled on to the upper part of the leg at nearly a right angle. The soft and pulpy-looking bone was quite exposed. The odour was very offensive. The foot was very odematous, and sensation was diminished. The femoral glands were not enlarged.

Oct. 31st. - After a consultation it was decided to amputate, which Mr. Emson accordingly did. Esmarch's elastic bandage and tourniquet was applied. Antero-posterior flaps were made, and the bones were divided about one inch below the tubercle of the tibia. The tissues were excessively soft, almost pulpy. Hæmorrhage was very slight. From the degenerated condition of the vessels secondary hxomorrhage was feared, and a tourniquet was accordingly placed in readiness over the femoral artery. She rallied exceedingly well. Evening temperature $101^{\circ}$.

On Nov. 1st there was a slight oozing of blood, about two ounces, during the twenty-four hours. The patient was comfortable. Temperature $100 \cdot 2^{\circ}$; pulse 102 . Had a quinine and iron mixture three times a day, and an opiate to relieve pain. Diet consisted of milk, three eggs, and an ounce of brandy. On the 2 nd the discharge was more sanious. Temperature $100^{\circ}$; pulse 114 , intermittent. She slept fairly well ; was very despondent. Ordered ten drops of the sedative solution of opium when pain was severe. On the 4th the wound was suppurating freely. She slept fairly well, and was more cheerful. Temperature $99^{\circ}$; pulse 102. Bowels open. On the 9th she was doing very well. She had a small bedsore over the sacrum, also one
over the right great trochanter. Temperature $99^{\circ}$. On the 17th temperature rose to $100^{\circ}$ morning, $99^{\circ}$ night. The bedsores, which had increased in size to that of a crown-piece, were painted with equal parts of collodion and castor oil. The stump looked well. All ligatures were taken away but one. From this time up to Dec. 2lst there was a gradual improvement both in the stump and bedsores, which latter were completely healed over. The temperature from this time to the 30th was between $98.4^{\circ}$ and $99^{\circ}$.

On Jan lst the patient was about the ward with crutches, but could not make very free use of them. On the 13th there were two small spots on the cicatrix of the stump not healed over, abont as large as a shirt-button. She was discharged on Jan. 17th, the stump having completely healed.

The advanced age of the patient and the uninterrupted progress of the case make it unusually interesting. The dressings throughout consisted of terebene dissolved in olive oil (1 in 20). Since her discharge she met with a slight accident by falling into the fender while attempting to remove a kettle from the fire, and scalded her hand as well as bruised her stump, but has completely recovered.

\section{PROVINCIAL MEDICAL SOCIETIES.}

Bradford Medico-Chirurgical Society.-June meeting; R. H. Meade, Esq., F.R.C.S., President, in the chair. Dr. GOYDER exhibited an Ovarian Tumour removed by $\mathrm{Mr}$. Spencer in the wards of the Bradford Infirmary on June 1st. The interesting facts in the case were that the tumour consisted of two parts : an upper, harder portion, consisting of a multitude of small cysts, so condensed as to appear solid; and a lower, large, single cyst, filled with the usual fluid. The woman presented herself at the out-patient department, under Mr. Goyder, for a fluctuating abdominal swelling, which, under treatment, greatly diminished, bringing to light a hard tumour, fixed and immovable, in the left hypochondrium. Subsequently the fluctuating swelling increased, and the patient was admitted into the house. A consultation, by Mr. Spencer, Dr. Bell, and Dr. Goyder, was held, and the fluctuating swelling pronounced to be ovarian, but the character of the hard portion was undetermined. After tapping and some weeks' interval the operation was performed successfully; the tumour was found to be as stated above, but the hardened portion was fixed by inflammatory adhesions to the abdominal wall at the left hypochondrium, and with some difficulty removed; this fact had rendered the diagnosis at first obscure. - The President cited a similar case in which the tumour was movable except at its upper part, where a hardish substance, in size and shape like a trencher, was immovably fixed, and seemed separable from the movable part; yet on operation it was found to be part of the ovarian growth, but fixed by adhesions, as in the former case.-Mr. Foster detailed a case of Tetanus. Patient, aged forty-eight, had sustained a rent-like wound, six inches long, over the left patella. By interrupted suture the wound healed in a week. On the eighteenth day after the accident he struck the knee against his bed, complained of pain, and said he had also a cold and sore-throat. On the nimeteenth day he developed difficulty of swallowing, stiffening of the masticatory, facial, and zygomatic muscles. On the twentieth day the injured leg, the muscles of the back and abdomen, were rigid; this state increased and persisted till death, on the twenty-ninth day. The pulse varied from 120 to 140. The treatment was by hypodermic injections of morphia and large doses of bromide of potassium and strong liquid nourishment. Mr. Foster said the case was a typical one. There seemed no cause of irritation in the wound itself, which healed perfectly. He then discussed the alliance and differentiation of this disease with hydrophobia and convulsions, and spoke of the view of its origin in injury to nervous fibres implicated in the wound. - Dr. Goyder attributed the origin of this disease to laceration, possibly of ligament as well as nerve-fibre. He said experience dictated to him in excessive laceration of fingers rather to amputate than risk tetanus by attempts to heal. He gave cases in illustration.-Mr. Mossop concurred that laceration of tissue was the exciting cause of tetanus. He cited the case of the death of his father from tetanus, coming on eleven days after laceration of the leg by a hook. He died on the seventeenth, erysipelas supervening.-Mr. Miall did not think that crushed fingers should be amputated. He had saved many by careful strapping; he had not seen enough of tetanus to make it a motive for amputation.-Dr. Whalley had seen one death from tetanus due to laceration of forearm, in which opium failed. The mischief seemed due to 